\title{
Venous Lakes of the Lips Successfully Treated by a Single Session of Multiplex Dual-Wavelength Laser
}

\author{
Seok Hwan Kim \\ Jin Young Kim \\ Eun Soo Park
}

Department of Plastic and Reconstructive Surgery, Soonchunhyang Univsersity Bucheon Hospital, Bucheon, Korea
Received May 7, 2016

Accepted May 10, 2016

\section{Correspondence}

Eun Soo Park

Department of Plastic and Reconstructive Surgery, Soonchunhyang Univsersity Bucheon Hospital, 1174, Jung-dong, Wonmi-gu, Bucheon 14584, Korea

Tel: +82-32-621-5319

Fax: $+82-32-621-5016$

E-mail: peunsoodaschmc.ac.kr

(C) Korean Society for Laser Medicine and Surgery

(c) This is an open access article distributed under the terms of the Creative Commons Attribution NonCommercial License (http://creativecommons.org/ licenses/by-nc/4.0) which permits unrestricted noncommercial use, distribution, and reproduction in any medium, provided the original work is properly cited.
Venous lakes of the lip is a common vascular lesion caused by a localized vascular dilatation that often occurs on sun-damaged skin. They are usually asymptomatic and uncomplicated, however treatment can be administered to improve cosmesis and occasionally to prevent bleeding. A 48-year-old female patient sought cosmetic removal of dark blue papules on the left lower lip and right upper lip which had been presented for several years. The patient was treated using the combination of pulsed-dye laser and long-pulsed Nd:YAG laser under topical anesthesia. Photographic documentation was performed before and after treatment. After a single treatment, the lesion disappeared completely. The patient tolerated the treatment well with minimal pain. Healing was completed within a week leaving an unnoticeable scarring without adverse effects including pigmentation, atrophy, wrinkling, and bleeding. The combination of pulsed-dye laser and long-pulsed Nd:YAG laser was highly effective and safe for the treatment of venous lakes. In an appropriate setting, excellent therapeutic and cosmetic results can be achieved in a single treatment session.

\section{Key words}

Venous lake; Pulsed dye laser; Nd:YAG laser 


\section{INTRODUCTION}

Venous lakes of the lip are common vascular lesions caused by a localized vascular dilatation that often occurs on sun-damaged skin. Histologically, they consist of dilated venules composed of a thin layer of endothelial cells with a thick wall of fibrous tissue located in the upper dermis. The lesion is thought to occur as a result of deterioration in the connective tissue in the vascular adventitia as well as in the dermis. 'They are commonly situated on the lower lip of elderly people. Venous lakes present as sharply demarcated, soft, dark blue to dark purple, round or oval nodules with a smooth surface. Although it is usually asymptomatic, but it can bleed after minor trauma. The clinical course of venous lakes is uncomplicated, but treatment may be demanded for cosmetic purpose or because of recurrent bleeding.

Numerous treatment modalities including cryosurgery, carbon dioxide laser, pulsed-dye laser, infrared coagulator, intense pulsed light, and sclerotherapy have been described. ${ }^{2-9}$ Treatment using different lasers has been used with variable success rates and specific complications. The pulsed-dye laser for the treatment of venous lakes has lower risk of scarring than with other types of lasers. But the results of pulsed-dye laser treatment for venous lakes were variable and demanded multiple sessions. ${ }^{10}$ Long-pulsed Nd:YAG laser has been reported effective for the vascular lesions on the face and neck. ${ }^{11}$ To our knowledge, there has not been a report of a case using two different lasers for venous lakes sequentially. Herein, we present a case of venous lakes on the upper and lower lip treated by the combination of pulsed-dye laser and long-pulsed Nd:YAG laser.

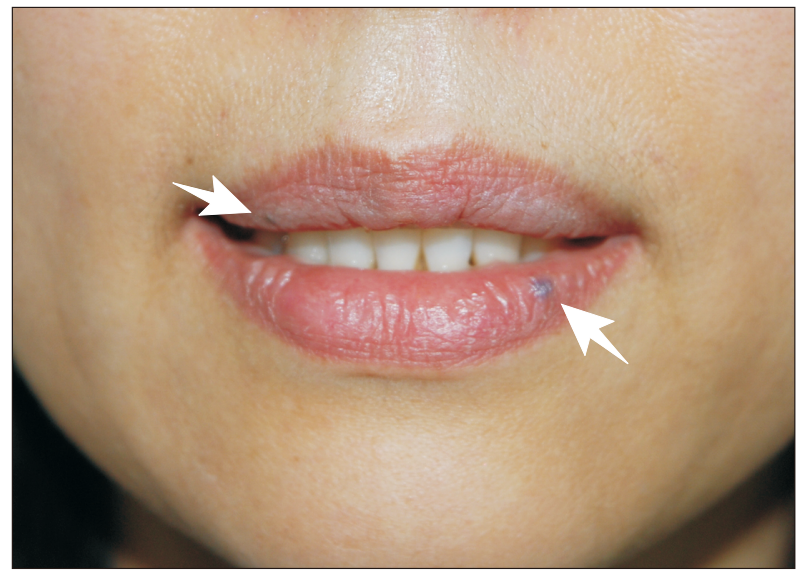

Fig. 1. Small venous lakes on the left lower lip and the right upper lip prior to treatment.

\section{CASE REPORT}

A 48-year-old female patient desired cosmetic removal of dark blue papules on the left lower lip and right upper lip which had been presented for several years. She had no other treatments prior to visiting our department. The treated lesion was $2 \mathrm{~mm}$ in diameter on the right upper lip and $4 \mathrm{~mm}$ in diameter on the left lower lip (Fig. 1). No similar lesions were noted on other areas of her body.

The patient was treated using the combination of pulsed-dye laser and Nd:YAG laser (Cynergy with MultiPlex; Cynosure, Westford, MA, USA) which allows the sequential emission of each wavelengths. The $595 \mathrm{~nm}$ pulsed-dye laser was fired first with a pulse duration of $0.5 \mathrm{~ms}$ and fluence of $4 \mathrm{~J} / \mathrm{cm}^{2}$. The long-pulsed $1064 \mathrm{~nm}$ $\mathrm{Nd}$ :YAG laser was fired later at $15 \mathrm{~ms}$ pulse, $40 \mathrm{~J} / \mathrm{cm}^{2}$ using a 7-mm spot size with a medium delay between the two pulses. The patient underwent a single session of laser treatment.

Topical lidocaine cream (EMLA; AstraZeneca Canada Inc., Ontario, Canadal was applied 60 minutes before treatment. After the laser treatment, topical antibiotic ointment (mupirocin) was applied to the wound without an additional occlusive dressing. Clinical photographs were taken before and after treatment. Response was assessed after 3 months. The treated lesions were checked for degree of clearance of the lesion, any signs of scarring and textural changes.

The patient received only one session of laser treatment. Immediately after laser treatment, venous lakes got lightened and flattened with surrounding erythema. Slight swelling of the treated area developed immediately but lasted less than a day. The patient tolerated the treat-

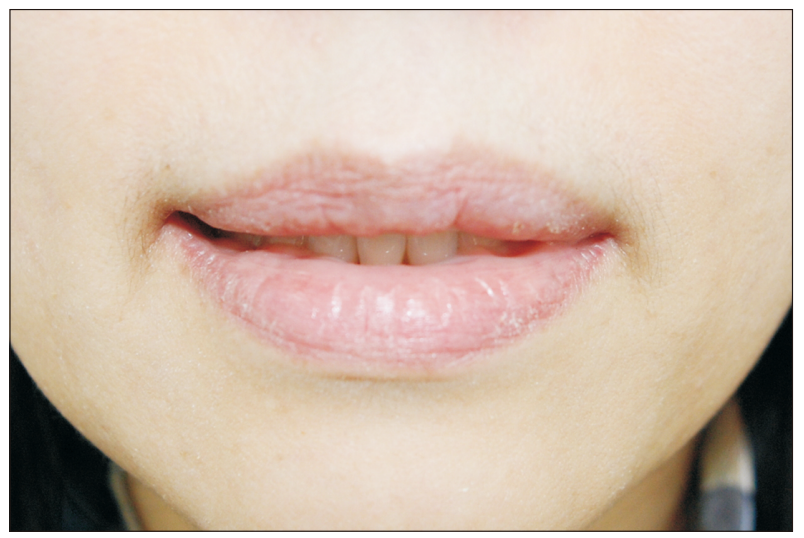

Fig. 2. 3-month follow-up after a single session of laser treatment. Note the normal color and texture of the upper and lower lip with complete resolution of venous lakes without scarring. 
ment well with minimal pain under topical anesthesia. The tissue sloughing was minimal and maintained only for a few days, and healing with reepithelization was completed within a week after treatment with no visible scarring. The cosmetic outcome was excellent, with complete clearance of venous lakes. No relevant secondary effects such as pigmentation or wrinkling were observed. No recurrence of the lesions was observed.

\section{DISCUSSION}

Venous lakes are relatively common on the face and especially around the mouth. Various treatment modalities treating venous lakes have been described in the literature including surgical excision, cryosurgery, electrosurgery, sclerotherapy, all regarded as effective by the authors. Different lasers have also been used such as the carbon dioxide laser, the diode laser, or the pulsed dye laser.

The pulsed-dye laser and the long-pulsed Nd:YAG laser have been used for years to treat a variety of vascular lesions The pulsed-dye laser induces a selective destruction of small cutaneous vessels, so it is considered to be the most effective device for treating smaller, more superficial vessels. The pulsed-dye laser has limitations because of the shallow depth of penetration. For the treatment of venous lakes, pulsed-dye lasers have resulted in minimal scarring and adverse effects, but repetitive sessions may be needed to achieve complete resolution. The longpulsed $1064 \mathrm{~nm}$ Nd:YAG laser provides greater depth of penetration but lower specificity. It penetrated deeply into skin and has greater affinity for deeper large vessels. Owing to deep penetration, the long-pulsed $\mathrm{Nd}$ :YAG laser has shown to be effective in treating venous lakes.

In the present study, we used a laser device combining a pulsed-dye laser with a Nd:YAG laser into one unit (Cynergy with MultiPlex; Cynosure, Westford, MA) which allows the sequential emission of each wavelengths. Vascular lesions are diverse with vessels of varying size, density, color, flow and depth. The use of dual sequential wavelengths has been studied as a method of minimizing the side effects of these lasers, while increasing the efficacy for treating vascular lesions. The pulsed-dye laser is fired first and absorbed by blood which converts hemoglobin to methemoglobin and thrombus. The Nd:YAG laser which fires later, is highly absorbed by these newly created methemoglobin and thrombus. The benefit of this process is the decreased background heating that allows the reduction of treatment fluence and reducing adverse effects while improving outcomes.

To our knowledge, there has been no report of a case using dual lasers for venous lakes sequentially so far. With sequential emission of two different wavelength and types of lasers, our case showed excellent therapeutic and cosmetic results with a single session without noticeable scarring and any relevant adverse effects.

In conclusion, based on our experience, the combination of pulsed-dye laser and long-pulsed Nd:YAG laser is safe and effective modality for the treatment of venous lakes resulting in excellent therapeutic and cosmetic outcomes in a single treatment session.

\section{ACKNOWLEDGMENTS}

This work was supported by the Soonchunhyang University Research Fund.

\section{REFERENCES}

1. Alcalay J, Sandbank M. The ultrastructure of cutaneous venous lakes. Int J Dermatol 1987;26:645-6.

2. del Pozo J, Peña C, García Silva J, Goday JJ, Fonseca E. Venous lakes: a report of 32 cases treated by carbon dioxide laser vaporization. Dermatol Surg 2003;29:308-10.

3. Kuo HW, Yang $\mathrm{CH}$. Venous lake of the lip treated with a sclerosing agent: report of two cases. Dermatol Surg 2003;29: 425-8.

4. Ah-Weng A, Natarajan S, Velangi S, Langtry JA. Venous lakes of the vermillion lip treated by infrared coagulation. $\mathrm{Br} J$ Oral Maxillofac Surg 2004;42:251-3.

5. Cecchi R, Giomi A. Pyogenic granuloma as a complication of cryosurgery for venous lake. Br J Dermatol 1999;140:373-4.

6. Jay H, Borek C. Treatment of a venous-lake angioma with intense pulsed light. Lancet 1998;351:112.

7. Majamaa $H$, Hjerppe M. Treatment of venous-lake angiomas with a carbon dioxide laser. J Eur Acad Dermatol Venereol 2003;17:352-3.

8. Suhonen R, Kuflik EG. Venous lakes treated by liquid nitrogen cryosurgery. Br J Dermatol 1997;137:1018-9.

9. Polla LL, Tan OT, Garden JM, Parrish JA. Tunable pulsed dye laser for the treatment of benign cutaneous vascular ectasia. Dermatologica 1987;174:11-7.

10. Cheung ST, Lanigan SW. Evaluation of the treatment of venous lakes with the 595-nm pulsed-dye laser: a case series. Clin Exp Dermatol 2007;32:148-50.

11. Scherer K, Waner M. Nd:YAG lasers $(1,064 \mathrm{~nm})$ in the treatment of venous malformations of the face and neck: challenges and benefits. Lasers Med Sci 2007;22:119-26. 